\title{
THE MAGNETOPAUSE ELECTRON LAYER ALONG THE DISTANT MAGNETOTAIL
}

\author{
D. N. Baker and E. C. Stone
}

California Institute of Technology, Pasadena, California, 91125

Abstract. An energetic electron layer is found immediately adjacent to, and outside of, the magnetopause surface along the distant magnetotail $\left(-40 \leqslant X_{S M} \leqslant-15 R_{F}\right)$. The layer has been detected using Caltech instrumentation aboard the earthorbiting spacecraft IMP-8 and is observed for electrons with energies $E \geqslant 200 \mathrm{keV}$. The present study shows that such electrons form a layer $\sim 3 R_{F}$ thick and are strongly streaming in a wellordered pattern, especially along the dusk magnetopause. The energy dissipation implied by the persistent flow may be a direct indication of nearly continuous magnetic merging at or near the magnetopause.

\section{Introduction}

Meng and Anderson (1970) first concluded that energetic electrons ( $E>40 \mathrm{keV}$ ) formed a layer at the magnetopause. HEOS-2 measurements (Page et al. 1973; Domingo et al., 1974, 1976) subsequently showed the magnetopause electron layer to be present in regions directly above the north geographic pole and showed that electrons in the layer frequently had energies up to 1 or $2 \mathrm{MeV}$. Domingo et al. found evidence for a net tailward flow of electrons of up to $10 \%$. Meng and Anderson (1975) found electrons ( $E>45 \mathrm{keV}$ ) in the polar cusp region to be isotropic in pitch angle (a1though anisotropies up to $30 \%$ might have been missed, according to the authors).

Using the Caltech Electron/Isotope Spectrometer (EIS) aboard the earth-orbiting spacecraft IMP-8, we have sought observational evidence of a magnetopause electron layer along the boundary of the distant magnetotail. The IMP-8 orbit is mildly elliptical ( $23 \mathrm{R}_{\mathrm{F}}$ by $46 \mathrm{R}_{\mathrm{E}}$ ) with an initial orbital inclination of $29^{\circ}$ and a 12.2 -day orbital period. The EIS detects electrons with energies $E \geqslant 200 \mathrm{keV}$ in a $1 \mathrm{~mm}$-thick solid state detector. Anisotropy information is provided by onboard sorting of events into eight sun-fixed $45^{\circ}$ sectors. A $3.2 \mathrm{mg} / \mathrm{cm}^{2}$ aluminized mylar window on the EIS telescope excludes all protons with energies $<1.2 \mathrm{MeV}$, and a thin (47 $\mu \mathrm{m})$ detector allows us to monitor proton intensities ( $E>1.3 \mathrm{MeV}$ ) and make appropriate corfections (see Baker and Stone 1976, and references therein). For the cases presented here, proton fluxes with $\mathrm{E}>1.3 \mathrm{MeV}$ result in a correction of $\leqslant 10 \%$ of the median number of events in the peak sector.

We identify magnetopause crossings by using plasma data (L.A. Frank, private communication) and magnetic field data (R.P. Lepping and N. F. Ness, private communication) obtained with other IMP-8 instrumentation.

Copyright 1977 by the American Geophysical Union

\section{Observations}

The observed bow shock and magnetopause crossings during one orbit are shown by dashed, curved lines drawn on the trajectory plot in Figure 1. Note that the spacecraft was very near the magnetopause boundary from late on day 343 until the latter portion of day 346 . We observed that at $\sim 0900$ UT on day 343 , the intensity of electrons (E $\geqslant 200 \mathrm{keV}$ ) rose sharply from a background flux of $\leqslant 10^{-1}\left(\mathrm{~cm}^{2}-\mathrm{sec}-\mathrm{sr}\right)^{-1}$ to $\geqslant 5\left(\mathrm{~cm}^{2}-\mathrm{sec}-\mathrm{sr}\right)^{-1}$. The spacecraft was at $\rho \sim 29 R\left(\rho=\sqrt{Y^{2}+z^{2}}\right)$ at the time of the flux increase (which was at $\Delta \rho$ $\sim 3 \mathrm{R}$ from the magnetopause if this surface is assumed stationary).

In the lower portion of Figure 1 is shown a shaded horizontal bar. This bar delineates the various plasma regions in which the spacecraft was located at a given time. When the spacecraft was within the magnetosheath (characterized by intense tailward flow of plasma ions) the bar is cross-hatched. The unshaded gaps in the central portion of the shaded bar correspond to times when the spacecraft was clearly within the plasmavold, high-tail lobe or within the 'plasma mantle' as described by Rosenbauer et al., (1975). Regions of an ambiguous character are shown with a dotted gray shading.

When the spacecraft enters the magnetotail, fluxes of electrons ( $\geqslant 200 \mathrm{keV}$ ) ordinarily diminish to near interplanetary background levels. Thus, in this case, the intensities ( $\geqslant 200 \mathrm{keV}$ ) fell to $\sim 10^{-1}\left(\mathrm{~cm}^{2}-\mathrm{sec}-\mathrm{sr}\right)^{-1}$ within the tail, but were typically 0.5 to $20\left(\mathrm{~cm}^{2}-\mathrm{sec}-\mathrm{sr}\right)^{-1}$ in the layer within several $R_{R}$ of the magnetopause. Geomagnetic conditions in this period ranged from mild1y to moderately disturbed (3-hour $\mathrm{Kp}$ indices 2 to $5^{+}$) with the daily $\mathrm{Kp}$ sums being $38^{-}$, $27,28^{-}, 26,27^{+}$, and 20 for days 343 to 348 .

The energetic electrons in the magnetopause layer are continually streaming. Thus, we fit the observed angular distribution according to $C(\phi)=C(1+S \cos (\phi-\Delta))$, where $\phi$ is the azimuthai (solar ecliptic) direction, $C_{0}$ is the spin-averaged count rate, $\Delta$ is the direction of the maximum intensity, and $S$ is the streaming amplitude.

In the lower portion of Figure 1 we have plotted the 24.6-minute average value of $\mathrm{S}$ in the form of a flow vector. A vector is shown only when $j(E \geqslant 200 \mathrm{keV})>0.5\left(\mathrm{~cm}^{2}-\mathrm{sec}-\mathrm{sr}\right)^{-1}$ and the peak sector intensity is at least two standard deviations above the spin-averaged intensity.

It is clear that the streaming amplitude was large and the direction of flow was well-ordered on day 343 on the dusk magnetopause. The anisotropy was typically 10: or 20:1 toward $\phi_{\mathrm{SE}} \sim 150$. It 

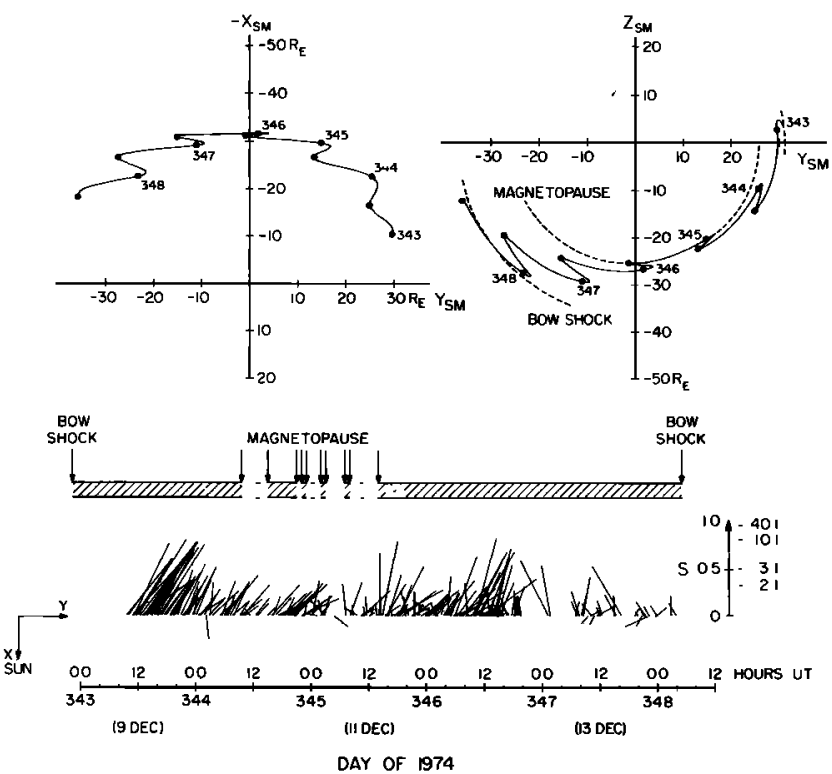

Figure 1. The upper portion of the figure shows the position of the IMP-8 spacecraft as projected onto the $\mathrm{X}-\mathrm{Y}$ and $\mathrm{Y}-\mathrm{Z}$ planes (in solar magnetospheric coordinates) for 9-14 December (days 343348) 1974. The lower portion of the figure indicates the observed streaming of electrons $(E \geqslant 200 \mathrm{keV}$ ) in the form of flow vectors projected onto the (solar ecliptic) X-Y plane. Gaps in the shaded horizontal bar shows periods when the spacecraft was inside the magnetopause as determined from plasma analyzer data. Ambiguous regions are shown dotted.

is also clear from the shaded bar that only outside this boundary was streaming (and the absolute intensity) large. The intense streaming persisted until $\sim 2000$ UT on day 346 at which time the spacecraft left the layer and intensities diminished $\sim 2$ orders of magnitude. Occasional brief bursts lasting tens of minutes to an hour were often seen nearer the bow shock late on day 347 and early on day 348 .

In Figure 2 are examples of the raw angular distribution data for several 24.6-minute intervals as were used to compute the flow vectors in Figure 1. These distributions tend to be sharply peaked, especially on the dusk side of the magnetotail and are aligned with the observed magnetic field. For the very sharply peaked distributions, the $(1+S \cos \phi)$ fit employed here underestimates the streaming amplitude but properly determines the direction of the flow. We have used the calibrated angular response of the detector system to compare with the sharply-peaked angular distributions such as shown on the left side of Figure 2. Such distributions are found to be consistent with an essentlally unidirectional beam of particles. Thus, for substantial periods of time, it appears that the energetic electrons have quite small pltch angles and move directly along the local field line.

In Figure 3 we show a second example of an IMP-8 high latitude magnetotail pass. During the central portion of this pass the spacecraft was in the plasma sheet (day 169) where electron fluxes were essentially isotropic, or in the high tail lobe (day 170) where absolute intensities were low. The 3-hour $\mathrm{Kp}$ indices ranged from $1^{+}$ to_ 4 in this period, with daily sums being 24,18 , $21^{-}$, and 26 for days 168-171.

The shaded bar in Figure 3 shows the rapid, rather well-defined passages through the magnetopause. The anisotropy was somewhat less than in Figure 1, but, especially on the dusk side, the flows, were well-ordered in direction toward $\phi_{\mathrm{SE}} \sim 150^{\circ}$, just as for the previous case.

Based upon examination of over two years of IMP-8 data, we conclude that energetic electron enhancements immediately adjacent to the tailward magnetopause are a nearly permanent feature of this region and are, in fact, part of the layer described by Meng and Anderson $(1970,1975)$ and Domingo et al. $(1974,1976)$. In a more extensive paper in preparation we show several other examples of strong electron flow in the magnetqpause layer and we show that the layer is present on almost every suitable cut through the highlatitude regions, suggesting that energetic particle acceleration and energy flow may also be nearly continual. These additional studies also show that absolute intensities in the layer have a noticeable overall dependence on geomagnetic activity as indicated by $\mathrm{Kp}$.
DAY 343: DUSK MAGNETOPAUSE LAYER

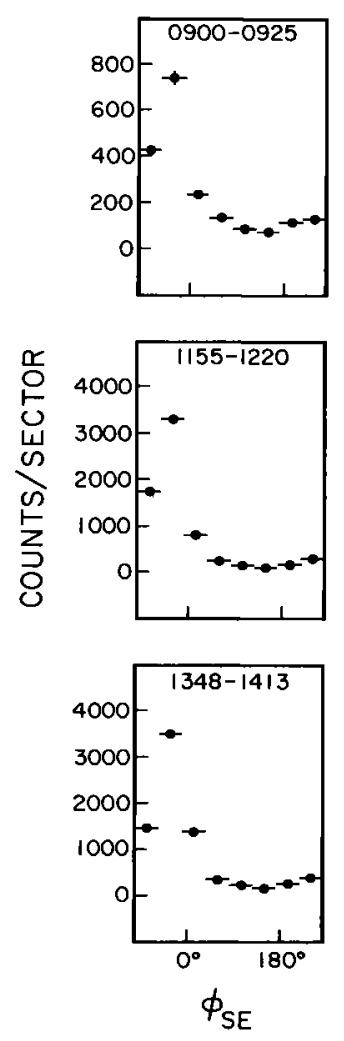

DAY 346: DAWN MAGNETOPAUSE LAYER
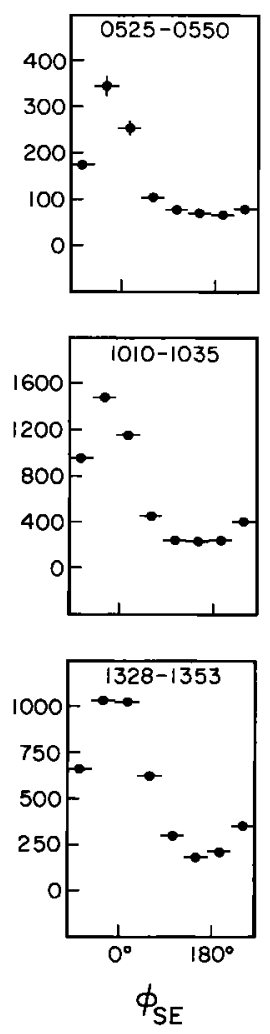

Figure 2. Examples of the raw angular distributions of electrons ( $\Rightarrow 200 \mathrm{keV}$ ) which were used to compute the flow vectors in Figure 1 . The time interval (UT) of the distribution is indicated above each example. Note the narrow-peaked character of the distributions, especially on the dusk side of the tail (day 343 ). 

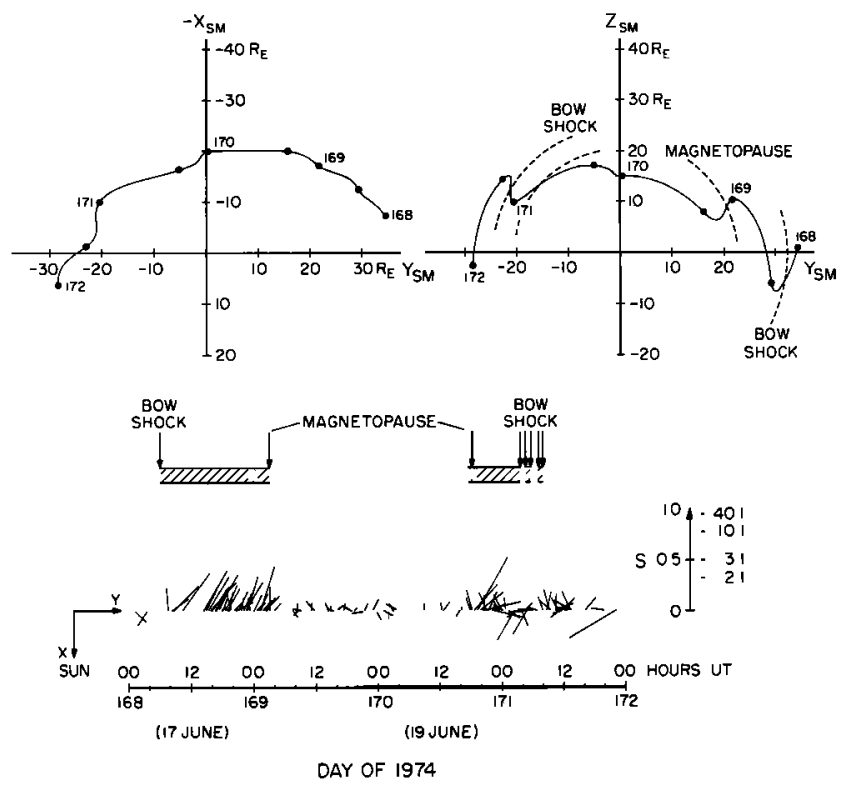

Figure 3. A figure similar to Figure 1 for a northern hemisphere pass through the magnetotail on 17-20 June (days 168-171) 1974 .

\section{Discussion}

Our observations appear to place several constraints on the source of the streaming electrons. First, we see the electron layer almost exclusively out side the magnetopause (as defined by both magnetic field and plasma flow criteria) of the distant tail. Second, we see high energy electrons to be continualiy streaming with no evidence of a significant return flow, suggesting a very short residence lifetime for electrons on a given field line near the magnetopause $(\sim 10 \mathrm{sec}$, or less). This demands a continuous source of energetic particles on, or near, the field lines which are draped over the magnetotail surface.

Figure 4 summarizes our observations. The upper portion of the figure illustrates that the electron layer is seen in the $\mathrm{Y}-\mathrm{Z}$ plane crosssection to be annular and all of our data suggest it is typically $\sim 3 R_{\text {in }}$ in thickness. The annular area of the layer is therefore computed to be $A_{L}$ $\sim 2 \times 10^{20} \mathrm{~cm}^{2}$.

Using all periods on days 343-346 when the plasma analyzer indicated that the spacecraft was in the magnetosheath (see the shaded bar in Figure 1) and using 4.1-minute ayerages, we obtained the distribution of the number of samples with a given counting rate in the peak sector (cf. the unshaded histogram in Figure 4). Approximately $80 \%$ of the samples show streaming, as indicated by the shaded histogram in Figure 4 which is the distribution of samples characterized by significant tailward flow with the peak sector rate at least $3 \sigma$ above the spin-averaged rate. The median number of counts is $\sim 70$. A similar distribution is found for the case shown in Figure 3 .

Cone angle effects are undoubtedly important in many of these measurements so that we may often fail to sample pitch angles near $0^{\circ}$, hence failing to observe a beamed flux of particles. To calculate a lower limit to the flux, however, we assume all pitch angles are observed and we take the detector response function appropriate for unidirec- tional fluxes to calculate the beam intensity values shown by the upper scale in Figure 4 . This method underestimates the fluxes in broader distributions such as shown on the right side of Figure 2. The computed unidirectional fluxes range from $\sim 1$ to $\sim 60 \mathrm{~cm}^{-2} \mathrm{sec}^{-1}$ flowing through A (with a mean value $\mathrm{J} \sim 5 \mathrm{~cm}^{-2} \mathrm{sec}^{-1}$ ): Using a typically observed energy spectrum $\left(\frac{d j}{d E} \propto E^{-3}\right)$, we get the average measured electron $\mathrm{dE}_{\text {energy to }}$ be $\langle E\rangle \sim 400 \mathrm{keV}$. Thus, the observed energy flow through $\mathrm{A}_{\mathrm{L}}$ is :

$$
1.3 \times 10^{14} \leqslant J A_{L}\langle E\rangle \leqslant 7.8 \times 10^{15} \mathrm{erg} \mathrm{sec}^{-1}
$$

for $\mathrm{E} \geqslant 200 \mathrm{keV}$.

Using the assumed spectrum to scale the results downward in energy to $\sim 10 \mathrm{keV}$ (and assuming the streaming persists down to such energies) we get:
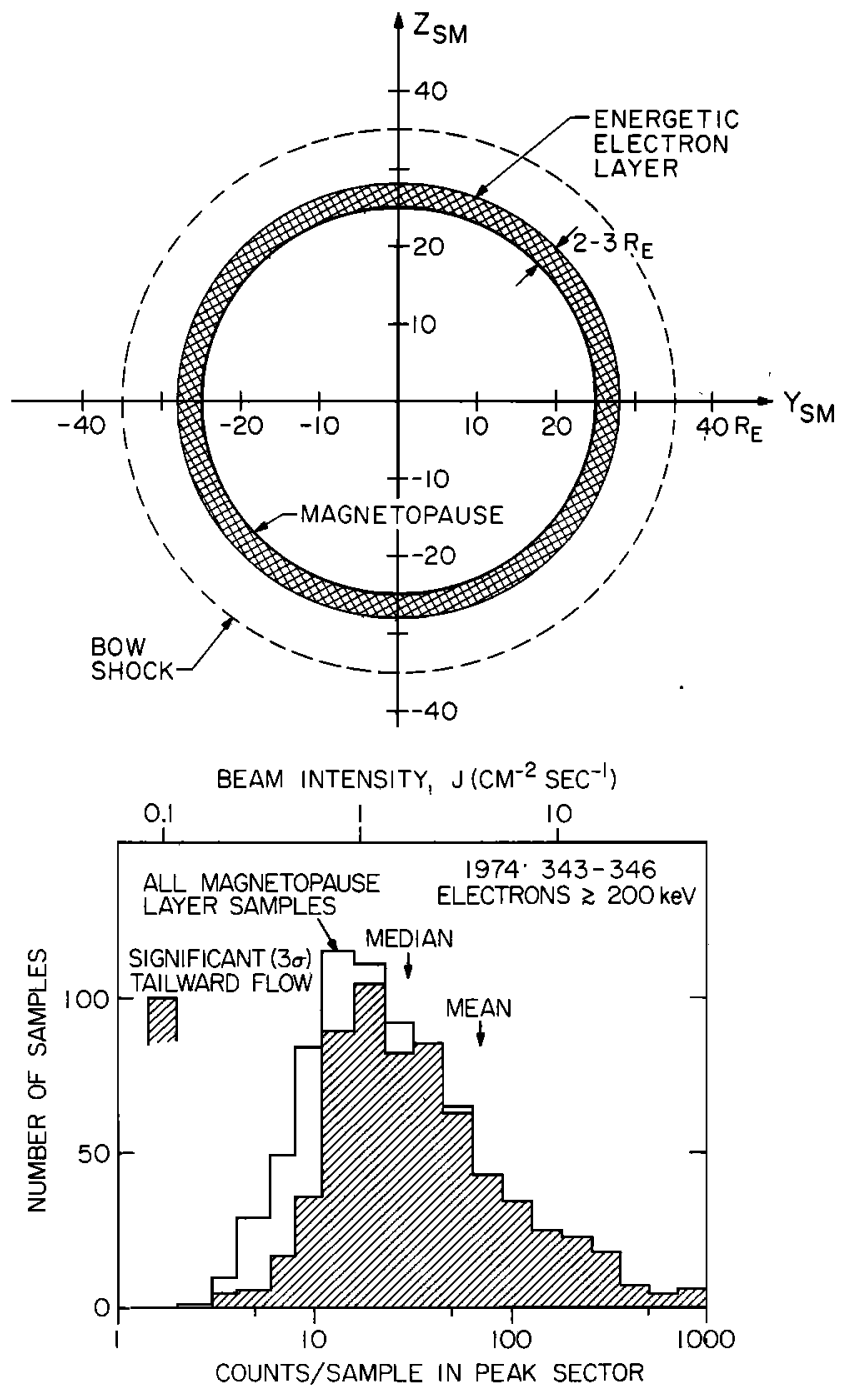

Figure 4. A summary of the characteristics of the magnetopause energetic electron layer at $-40 \leqslant \mathrm{X}_{\mathrm{SM}} \leqslant-15 \mathrm{R}_{\mathrm{F}}$. In the $\mathrm{Y}-\mathrm{Z}$ plane, the layer has an annular cróss-section 2-3 $R$ in thickness, as indicated by the cross-hatched region outside the magnetopause. The histogram in the lower portion illustrates the distribution of the number of samples exhibiting a given number of counts in the peak sector for the magnetopause layer shown in Figure 1. 


$$
3 \times 10^{15} \leqslant \mathrm{~J} \mathrm{~A}_{\mathrm{L}}\langle\mathrm{E}\rangle\left\langle 2 \times 10^{17} \mathrm{erg} \mathrm{sec}^{-1}\right.
$$

for $\mathrm{E} \geqslant 10 \mathrm{keV}$.

The energetic electron flow vectors, such as shown in Figure 1, appear consistent with a source near the nose or cusp of the magnetosphere or else on the dawn side of the tail. Although many geophysical processes may be responsible for the particle flow, the amount of energy which we observe to be flowing tallward along the magnetopause appears to be quite significant, particularly if our extrapolation to lower energies is at all valid. It may be that the energy flow we observe is due to dissipation associated with field line merging (connection) at the dayside magnetopause. Heikkila (1975) has concluded that $\sim 10^{18}$ ergs sec -1 should be evident as an energized plasma along the downstream magnetopause if merging is, indeed, proceeding at the front of the magnetosphere. As noted by Axford (1976), the tailward magnetopause electron layer could be conveniently explained on this basis and the estimated energy flows we have made above would constitute a sizable fraction of the total expected energy dissipation for the dayside merging process.

On the other hand, Axford (1976) has also shown that field line reconnection in the magnetotall should provide energy at a rate comparable to that available from dayside merging $\left(\sim 10^{18}\right.$ ergs $\mathrm{sec}^{-1}$ ). Thus, it may be that the energy flow we observe is that due to tailward merging. Indeed, at low latitudes along the dawn flank of the magnetotall we see intensities, angular distributions, and energy spectra for electrons $(E \geqslant 200 \mathrm{keV})$ which suggest that local acceleration may be proceeding in that region as suggested by Frank et al. (1976). In any case, it appears that the energization is nearly continual and that the field lines carrying this particle flow are external to the magnetopause. Further study, perhaps with several spacecraft, may further define the source supplying the magnetopause layer.

\section{Acknowledgments}

We thank L. A. Frank both for providing us with IMP-8 LEPEDA energy-time spectograms and for many helpful discussions. We are also grateful to R. P. Lepping and N. F. Ness for providing us with the IMP-8 magnetometer data through $\mathrm{J}$. H. King of the National Space Science Data Center. We are grateful to $R$. E. Vogt who has been close-
Iy involved in this investigation and to $W$. E. Althouse, G. J.Hurford, J. E. Lupton, and R. A. Mewaldt, who made significant contributions to various phases of this program. This work was supported in part by the National Aeronautics and Space Administration under contract NAS511066 and Grant NGR 05-002-160.

\section{References}

Axford, W. I., Flow of mass and energy in the solar system, Physics of Solar Planetary Environments, Proceedings of ISSTP, p. 270 , edited by D. J. Williams, Amer. Geophys. Union Publication, 1976.

Baker, D. N. and E. C. Stone, Energetic electron anisotropies in the magnetotail: identification of open and closed field lines, Geophys. Res. Letters, $\underline{3}, 557,1976$.

Domingo, V., D. E. Page, and K-P Wenzel, Energetic electrons at the magnetopause, in Correlated Interplanetary and Magnetospheric Observations, edited by D. E. Page, p. 159, D. Reidel, Dordrecht, The Netherlands, 1974.

Domingo, V., D. E. Page, and K-P Wenzel, Energetic and relativistic electrons near the polar magnetopause, ESLAB Res. Rep. 76/09, 1976.

Frank, L. A., K. L. Ackerson, and R. P. Lepping, On hot tenuous plasmas, fireballs, and boundary layers in the earth's magnetotail, J. Geophys. Res. 81, 5859, 1976.

Hetkkila, W. J., Is there an electrostatic field tangential to the dayside magnetopause and neutra1 line?, Geophys. Res. Letters, 2, 154 , 1975.

Meng, C-I., and K. A. Anderson, A layer of energetic electrons ( $>40 \mathrm{keV}$ ) near the magnetopause, J. Geophys. Res., 75, 1827, 1970.

Meng, C-I., and K. A. Anderson, Characteristics of the magnetopause energetic electron layer, J. Geophys. Res., 80, 4237, 1975.

Page, D. E., V. Domingo, D. Kohn, B.G. Taylor, K-P Wenzel, and P. C. Hedgecock, High energy electrons at the magnetopause above the north pole, Space Res. XIII, 631, 1973.

Rosenbauer, H., H. Grunwaldt, M. D. Mont gomery, G. Paschmann, and N. Sckopke, HEOS2 plasma observations in the distant polar magnetosphere: The plasma mantle, J. Geophys. Res. 80 , 2723, 1975.

(Received January 17, 1977; revised February 15, 1977 ; accepted March 7, 1977.) 